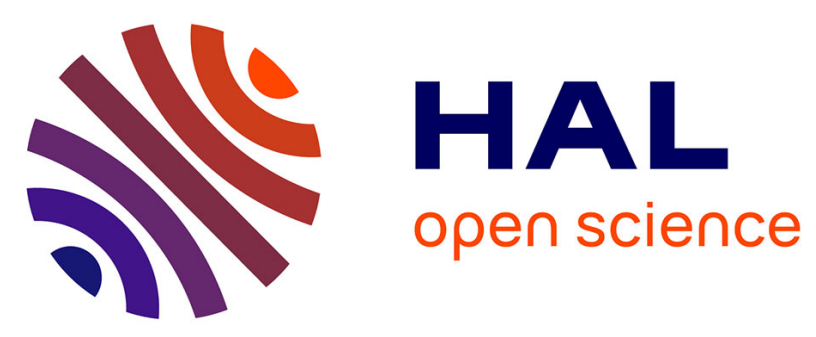

\title{
Patient dose evaluation in computed tomography: A French national study based on clinical indications
} B. Habib Geryes, A. Hornbeck, V. Jarrige, N. Pierrat, H. Ducou Le Pointe, S. Dreuil

\section{- To cite this version:}

B. Habib Geryes, A. Hornbeck, V. Jarrige, N. Pierrat, H. Ducou Le Pointe, et al.. Patient dose evaluation in computed tomography: A French national study based on clinical indications. Physica Medica, 2019, 61, pp.18-27. 10.1016/j.ejmp.2019.04.004 . hal-02524213

\section{HAL Id: hal-02524213 \\ https://hal.science/hal-02524213}

Submitted on 27 May 2020

HAL is a multi-disciplinary open access archive for the deposit and dissemination of scientific research documents, whether they are published or not. The documents may come from teaching and research institutions in France or abroad, or from public or private research centers.
L'archive ouverte pluridisciplinaire HAL, est destinée au dépôt et à la diffusion de documents scientifiques de niveau recherche, publiés ou non, émanant des établissements d'enseignement et de recherche français ou étrangers, des laboratoires publics ou privés.

\section{(ㅇ)(1) $\$$}

Distributed under a Creative Commons Attribution - NonCommercial - NoDerivatives| 4.0 


\section{Patient dose evaluation in computed tomography: a French national study based on clinical indications}

\section{Authors names and affiliations:}

Bouchra Habib Geryes $^{\mathrm{a}, \mathrm{c}}$ (equal contribution, joint first author), Amaury Hornbeck ${ }^{\mathrm{a}, \mathrm{b}}$ (equal contribution, joint first author), Valérie Jarrige ${ }^{\mathrm{a}, \mathrm{d}}$, Noëlle Pierrat ${ }^{\mathrm{a}, \mathrm{e}}$, Hubert Ducou Le Pointe ${ }^{\mathrm{f}, \mathrm{g}}$, Serge Dreuil ${ }^{\mathrm{a} h}$

a. French Society of Medical Physics (SFPM), Centre Antoine Béclère, 47 Rue de la Colonie, 75013 Paris, France

b. Department of Medical Physics, ALARA Expertise, 5 Allée de l'Europe, 67960 Entzheim, France

c. Department of Adult Radiology, Necker Enfants Malades University Hospital, 149 rue de Sèvres 75743 Paris Cedex 15, France

d. Department of Radiology, Jacques Coeur Hospital, 145 Avenue François Mitterrand, 18000 Bourges, France

e. Department of Radiology, Curie Institute, 26 Rue d'Ulm, 75005 Paris, France

f. French Society of Radiology (SFR), 47 Rue de la Colonie, 75013 Paris, France

g. Department of Paediatric Radiology, Sorbonne University, Trousseau Hospital, APHP, 26 Avenue du Dr Arnold Netter, 75012 Paris, France

h. Institut de Radioprotection et de Sûreté Nucléaire, 31 avenue de la Division Leclerc, B.P. 17, 92262 Fontenay-aux-Roses Cedex, France

Corresponding author: Serge Dreuil, E-mail: serge.dreuil@irsn.fr, Telephone: +33 15835 80 20, Fax: +33158358829

Declarations of interest: none 


\begin{abstract}
Purpose. A national survey was performed to assess patient dose indicators based on clinical indication and on patient morphology for most common adult computed tomography (CT) examinations in France.
\end{abstract}

Methods. Seventeen groups of clinical indications (GCIs) for diagnostic CT in adult patients were considered based on their frequency and on image quality requirements. Data was collected for 15-30 consecutive examinations performed between 2015 and 2017, per CT scanner and GCI. Distributions of total examination Dose-Length Product (DLP) and Volume CT Dose Index $\left(\mathrm{CTDI}_{\mathrm{vol}}\right)$ were assessed for each GCI as a function of patient gender or patient Body Mass Index (BMI) for head/neck and trunk examinations, respectively. Results. 6610 examinations were analysed. Median total exam DLP values were higher for men compared to women patients for head and neck examinations: difference ranged from $6 \%$ for ear trauma indication ( 577 vs $543 \mathrm{mGy} \cdot \mathrm{cm}, \mathrm{p}=0.01$ ) to $35 \%$ for brain tumour GCI (1472 vs $1093 \mathrm{mGy} \cdot \mathrm{cm}, \mathrm{p}<0.01)$. For trunk examinations, total exam DLP increased consistently with patient's BMI. For normal-BMI patients, median CTDI $_{\mathrm{vol}}$ and DLP differed significantly between different GCIs for single-phase CT of the chest ( $3 \mathrm{mGy}$ and $112 \mathrm{mGy} \cdot \mathrm{cm}$, respectively, for chronic obstructive pulmonary disease group vs $5.8 \mathrm{mGy}$ and $207 \mathrm{mGy} \cdot \mathrm{cm}$ for pulmonary embolism group, $\mathrm{p}<0.05)$ and of the abdomen-pelvis $(5.6 \mathrm{mGy}$ and 284 $\mathrm{mGy} \cdot \mathrm{cm}$, respectively, in renal colic group vs $9.5 \mathrm{mGy}$ and $463 \mathrm{mGy} \cdot \mathrm{cm}$ in occlusive syndrome group, $\mathrm{p}<0.05)$.

Conclusion. This study provides morphological- and clinical-based patient dose indicators in CT as a practical tool for clinical practices optimisation. 
Keywords: Computed Tomography; Clinical Diagnostic Reference Levels; Radiation Protection; Nationwide Survey.

\section{Abbreviations and acronyms:}

AP: Abdomen and Pelvis

BMI: Body Mass Index

CAP: Chest Abdomen and Pelvis

COPD: Chronic Obstructive Pulmonary Disease

CT: Computed Tomography

$\mathrm{CTDI}_{\mathrm{vol}}$ : Volume Computed Tomography Dose Index

DACS: Dose Archiving and Communication System

DLP: Dose-Length Product

DRL: Diagnostic Reference Level

FBP: Filtered Back Projection

GCI: Group of Clinical Indications

ICRP: International Commission on Radiological Protection

IR: Iterative Reconstruction algorithm

NDRL: National DRL

PE: Pulmonary Embolism

SFPM: French Society of Medical Physics

SFR: French Society of Radiology

USA: United States of America 


\section{Introduction}

Computed tomography (CT) is a powerful clinical tool enabling faster and more accurate diagnosis than interventional techniques or conventional plain radiography. However, some epidemiological studies have associated CT during childhood with an increased risk of radiation-induced malignancies [1-4]. Even if the outcomes of these studies are still controversial [5-7], a sensible use of the modality is of common sense. It requires strict adherence to the principles of radiation protection - justification and optimisation of the patient radiation exposure [8]. At the core of the optimisation principle is the establishment of diagnostic reference levels (DRLs), first proposed by the International Commission on Radiological Protection (ICRP) [9] and subsequently introduced into European legislation [10-11]. DRLs are suggested action levels above which a facility should review its methods and determine if acceptable image quality can be achieved at lower doses. An approach to establishing DRLs in CT had been proposed by ICRP [12-13], using two primary metrics: Dose-Length Product (DLP) and Volume Computed Tomography Dose Index (CTDI vol $_{\text {). }}$ National DRLs (NDRLs) in CT have been established in many countries [14-22]. Most of these NDRLs are defined for a single acquisition (i.e. phase), a standard patient morphology and are based on an anatomical region $[15,18,21]$. However it is evident that patient radiation dose depends on the number of acquisitions and on the patient's morphology. Likewise, patient radiation dose depends on the clinical indication, as image quality requirements are directly determined by the clinical purpose of the examination. This has been acknowledged, at least in part, by some NRDLs systems [13, 14, 16, 17, 19, 20, 22]. However, none of these NDRLs studies has simultaneously involved a large number of examinations, clinical indications and patient size.

The purpose of this national survey was to investigate current radiation doses for common clinical indications in adult CT examinations throughout France and to assess patient radiation 
dose as a function of patient characteristics. This scientific pilot study did not intend to establish French national DRLs but aimed at demonstrating that clinical indications as well as patient morphology need to be taken into account in the DRLs setting for better dose optimisation. The methodology and results proposed in this study could serve as an important input for the national radiation protection authorities to future updates of French DRLs, which would need larger subgroup sample sizes.

\section{Material and Methods}

The study conformed to scientific principles and national research ethics standards. Patients' care was not modified in any manner because of this study. Data were retrospectively collected by participants on their secured radiological information systems or on their institution's medical records. Data were sent to the investigators in an anonymous form. The investigators had no access to the participants' information systems and therefore had no possibility of reidentifying the patients. Consequently, informed consent of the patients was not required by personal data protection rules at the time of the study.

\section{Patient radiation dose survey}

A national retrospective survey was conducted by the French Society of Medical Physics (SFPM) on patients aged $\geq 16$ years who underwent CT examinations in France between 2015 and 2017. A list of 22 groups of clinical indications (GCIs) for diagnostic CT in 9 anatomical regions was initially defined by the SFPM, with the collaboration of the French Society of Radiology (SFR) and its associated organ sections, based on the frequency and the image quality requirements of the clinical indication. Seventeen GCIs were eventually included in the study (see Table 1 and next section for more details). A national call for volunteers for one or more GCIs was sent to the medical physicists' community. Data collection was performed 
by each participant independently, using one or more of the following means: picture archiving and communication system (80\% of the participants), radiological or hospital information system (61\%), dose archiving and communication system (50\%) or paper medical records $(9 \%)$. Anonymised data were transmitted to the investigators with the help of a preformatted Excel datasheet. For each participant's CT scanner and specific GCI, 15-30 consecutive examinations were required. Collected data focused on the complete examination with details of each acquisition. Collected data included CT scanner characteristics (manufacturer, model, commissioning year and maximum collimation); patient information (age, and, optionally, gender, weight and height); acquisition parameters (use of contrast media, and, optionally, acquisition type -axial or helical-, scan length, $\mathrm{kV}$, use of tube current modulation, use of patient restraint equipment and acquisition description); image reconstruction algorithm -Iterative Reconstruction (IR) or Filtered Back Projection (FBP) -; clinical information (clinical indication from the medical report and optionally, exam codification used for medical insurance) and dose data (CTDI ${ }_{\mathrm{vol}}$, DLP and phantom type $16 / 32 \mathrm{~cm}$ diameter- for each acquisition without localizer radiograph acquisitions and without acquisitions related to the contrast media tracking as well as total examination DLP).

\section{Exclusion criteria}

An essential point in this study was to have reliable data. Each participating facility was asked to check the clinical indication of the CT examination in the medical report. The working group of the SFPM and the SFR checked each collected examination data based mainly on the series description, contrast media use, phantom type $(16$ or $32 \mathrm{~cm})$ and/or scan length.

Examinations were excluded from the analysis based on the following criteria:

- Patient's age < 16 years. 
- Missing required information (i.e. clinical indication, CT scanner characteristics, use of contrast media, image reconstruction algorithm and dose information).

- Incoherent information, e.g. non correspondence between phantom type and body region or obvious mistypes.

- Incomplete examinations, i.e. missing one or more acquisition, as detected by comparison of the total examination DLP and the sum of all acquisition's DLP.

- Mixed indications, i.e. examinations not focusing exclusively on the initial clinical indication(s), such as extension assessments with respect to the normal practice protocol(s) of the considered GCI (e.g. brain exploration in ear trauma indication or abdominal exploration in pulmonary embolism indication). Even if these examinations correspond to the right initial clinical indication and to justified radiological practices, they could have introduced a bias in the calculation of the GCIs' dosimetric indices and were therefore excluded from the analysis.

Ultimately, sets of data counting less than 10 examinations per CT scanner and per GCI at the end of the exclusion process were excluded from the analysis. Likewise, GCIs having less than 10 contributing CTs were also excluded from the study. Consequently, 5 GCIs were abandoned (aortic dissection, suspicion of a lung cancer, screening for secondary liver cancer, suspicion of a kidney cancer and haematuria). Table 1 indicates the 17 GCIs retained for this study, together with the correspondent body part and the abbreviated name, which will be used thereafter in the paper.

\section{CT dose indices analysis}

Current CT scanners provide $\mathrm{CTDI}_{\mathrm{vol}}$ (in $\mathrm{mGy}$ ) and DLP (in $\mathrm{mGy} \cdot \mathrm{cm}$ ) for each acquisition, which are measured in 16 or $32 \mathrm{~cm}$ diameter acrylic phantoms [23]. Given their ease of collection, they were the main parameters selected for this study. By also taking into account 
the number of scan acquisitions used, total exam DLP is directly related to patient risk. Therefore, it may be used to set DRLs for CT-examinations [12-13] and hence was the primary parameter recorded in this study.

Total exam DLP based on the number of acquisitions per examination $n_{a}$ was analysed as a function of gender for head and neck GCIs. For trunk/body regions, a refined analysis per body mass index (BMI) groups was possible for 11 GCIs using the international classification of adult underweight $\left(\mathrm{BMI}<18.5 \mathrm{~kg} / \mathrm{m}^{2}\right)$, normal $\left(18.5 \leq \mathrm{BMI}<25 \mathrm{~kg} / \mathrm{m}^{2}\right)$, overweight $(25 \leq$ $\left.\mathrm{BMI}<30 \mathrm{~kg} / \mathrm{m}^{2}\right)$ and obesity $\left(\mathrm{BMI} \geq 30 \mathrm{~kg} / \mathrm{m}^{2}\right)[24]$.

Examinations characteristics $\left(\mathrm{kV}, \mathrm{mA}\right.$, image reconstruction type, $\mathrm{CTDI}_{\mathrm{vol}}$ and DLP) between different GCIs relative to the same body part were compared. This analysis was restricted to single-acquisition examinations and, for trunk examinations, to normal-BMI patients in order to overcome the influence of the number of acquisitions and the patient morphology on dose.

\section{Statistical analysis}

Statistical analysis was performed using R version 3.4.3 (R Foundation for Statistical Computing, Vienna-Austria). Categorical variables were expressed as percentages of the number of examinations, which was systematically indicated. Dose indices data were found to be non-normally distributed. Therefore, differences between median values were assessed using a non-parametric test (Wilcoxon rank-sum). The significance level was set to 0.05 . The maximum $\mathrm{p}$ value was quoted when more than two GCI subgroups were compared. Twenty-fifth, $50^{\text {th }}$ and $75^{\text {th }}$ percentile values of total exam DLP were calculated once on the distribution of pooled data for all facilities and once again, without BMI or $\mathrm{n}_{\mathrm{a}}$ stratification, on the distribution of median values per facility according to ICRP recommendations [13]. The two methods gave mean(min-max) differences of $14(1-41) \%, 7(0-16) \%$ and $9(1-24) \%$ in resulting $25^{\text {th }}, 50^{\text {th }}$ and $75^{\text {th }}$ percentiles of total exam DLP for each GCI, respectively. The 
mean differences being within or very close to the mandatory accuracy on displayed values of DLP $( \pm 20 \%)$, we chose to present only results of the first method with pooled data distribution for all sub-groups (examination DLP per GCI, per gender/BMI as a function of the number of acquisitions per examination $n_{a}$ ). This was possible because of the similar number of examinations submitted by all CT scanners, which gives them an equivalent weight.

Only descriptive comparisons were possible with published data from other countries because of the variability in methods among countries and of insufficient clinical or patient information in other studies for statistical comparisons.

\section{Results}

A total of 9667 acquisitions, corresponding to 7102 examinations included in the 17 selected GCIs, was collected from $88 \mathrm{CT}$ scanners in 53 facilities. After the data validation process, 6610 examinations (8620 acquisitions, 86 CT scanners, 53 facilities) were analysed. This included $24 \%$ chest, $21 \%$ head, $14 \%$ abdomen and pelvis (AP), $11 \%$ chest, abdomen and pelvis (CAP), $9 \%$ pelvis, $6 \%$ ear, $5 \%$ mandible/maxilla, $5 \%$ cervical spine and $5 \%$ body CTexaminations (Table 1). $87 \%$ of participating facilities were public hospitals (59\% academic and $41 \%$ non-academic), $9 \%$ comprehensive cancer care institutes and $4 \%$ private centres. All French regions were represented. Figure 1 shows the distribution of collected examinations by scanner manufacturer, commissioning year and GCI. The median commissioning year was 2013. Examinations were performed on CT scanners from different manufacturers: General Electric Healthcare (49\%), Siemens Healthineers (35\%), Philips (9\%) and Canon Medical Systems Corporation, formerly Toshiba Medical (7\%). Table 1 shows the distribution over the 17 GCIs of the number of centres, CT scanners and examinations collected and validated.

Mean number of acquisitions per examination $\left\langle n_{a}\right\rangle$ and clinical practices distribution in terms 
of $n_{a}$ value and contrast media use are also presented. $\left\langle n_{a}\right\rangle$ ranges from 1 (Pelviscan) to 3.67 (Polytrauma). Fifteen GCIs out of 17 gave a majority of single-acquisition CT examinations. Two GCIs had a majority of CT examinations with two acquisitions (Head/Tumour) or three acquisitions (Polytrauma). Fully unenhanced or fully contrast-enhanced examinations were dominant amongst all but the same two GCIs (Head/Tumour, Polytrauma) where mixed studies (i.e. including both unenhanced and enhanced acquisitions) were dominant.

Figure 2 shows the distribution of total examination DLP for all studied GCIs, independently of $n_{a}$ value and without patient grouping per gender or size. Median examination DLP varied from $25 \mathrm{mGy} \cdot \mathrm{cm}$ for Pelviscan to $3184 \mathrm{mGy} \cdot \mathrm{cm}$ for Polytrauma. Tables $2 \mathrm{a}$ and $2 \mathrm{~b}$ summarize total exam DLP interquartile ranges based on the number of acquisitions per examination $n_{a}$ and on patient gender for 6 head and neck GCIs or patient BMI for 11 trunk/body GCIs.

Examinations were evenly distributed between men and women patients (49.7\% men, 50.3\% women) and total exam DLP median values were higher in men patients for all head and neck GCIs. Median DLP difference ranged from 6\% in Ear/Trauma to 35\% in Head/Tumour, this difference being statistically significant for all head GCIs $(\mathrm{p}<0.01)$, for Ear/Trauma $(\mathrm{p}=$ $0.01)$, and Neck/Trauma $(p=0.03)($ Table 2a).

For trunk/body examinations (Table $2 \mathrm{~b}$ ), $45 \%$ of examinations were in normal-weight patients, $34 \%$ in overweigh, $15 \%$ in obese and $6 \%$ in underweight patients. Total exam DLP median values increased dependably with patient BMI for all GCIs and all $n_{a}$ sub-groups. In general for every GCI in any body part, total exam DLP increased consistently with the number of involved CT acquisitions, $n_{a}$ (Tables $2 \mathrm{a}$ and $2 \mathrm{~b}$ ). 
Table 3 shows the variation of single-acquisition examinations characteristics and dose indices between different GCIs relative to the same body part. Characteristics of unique GCIs (Ear/Trauma, Dental, Neck/Trauma, Pelviscan) are also summarized. Distribution of CTDI ${ }_{\mathrm{vol}}$ values and examination DLP are represented in Figure 3 for head GCIs (3a, 3c) and thorax, AP and CAP GCIs (3b, 3d). All GCIs but Ear/Trauma (33\%), Dental (16\%) and Pelviscan (23\%) examinations were mostly done with automatically modulated current (mA). IR algorithms were mostly used in all GCIs examinations except for AP/occlusion (50\% IR and $50 \%$ FBP). For head and neck examinations, $110 / 120 \mathrm{kV}$ were dominant in all GCIs except for Ear/Trauma where 130/135/140 kV were mostly used. For chest and AP examinations, kV values varied between $100 \mathrm{kV}$ and $110 / 120 \mathrm{kV}$. For the 2 GCIs in CAP examinations, 110/120 kV were dominant. For Head GCIs, Median CTDI ${ }_{\text {vol }}$ was slightly higher in $\operatorname{Head} /$ Hemo $(\mathrm{p}=0.03)$ and median examination DLP for Head/Tumour was lower $(\mathrm{p}=0.01)$ than the two other head GCIs. The analysis for chest examinations showed that both CTDI ${ }_{\mathrm{vol}}$ and examination DLP median values for Chest/PE were significantly higher than the other GCIs $\left(\mathrm{p}<10^{-3}\right)$ and significantly lower for Chest/COPD ( $\mathrm{p}=0.03$ and 0.01 for $\mathrm{CTDI}_{\mathrm{vol}}$ and DLP respectively). Every AP GCIs showed a significant difference from one another both in median $\mathrm{CTDI}_{\mathrm{vol}}(\mathrm{p}<0.01)$ and examination DLP $(\mathrm{p}=0.01)$. Lastly, no significant difference was observed between CAP/Tumour and CAP/Infectious.

\section{Discussion}

$\mathrm{CTDI}_{\text {vol }}$ (for single-acquisition) and total exam DLP (per number of involved acquisitions) national distributions were assessed on 17 GCIs as a function of patient gender or BMI for head and neck or trunk examinations respectively. For head and neck GCIs, total exam DLP values were higher for men compared to women patients, considering the same number of acquisitions within a same examination (Table 2a). This is likely due to larger size [25] and 
more dense head and neck of men compared to women's, especially when analysed examinations were mostly performed with automatically modulated current (Table 3). For all trunk/body GCIs, total exam DLP increased consistently with patient BMI for all examination groups (Table 2b). Thus, our results confirmed the strong dependence of dose estimators on patient morphology.

When comparing different GCIs within same body region, CTDI ${ }_{\mathrm{vol}}$ and DLP for singleacquisition examinations showed significantly higher values for Chest/PE compared to other GCIs in chest region. This can be explained by the high image quality requirements for this indication, reflected in CT examination characteristics mostly performed with contrast media in Chest/PE, compared to other GCIs (Table 1). Conversely, the lower dose indices values observed for Chest/COPD are related to the lower image quality requirement of this indication that is always performed without contrast media and takes advantage of the high natural contrast of the lung parenchyma. The analysis for AP examinations showed significantly lower values for AP/Colic compared to other GCIs. Lower dose protocols mostly performed without contrast enhancement (Table 1) and lower kV (mostly $100 \mathrm{kV}$ ) (Table 3) were used in AP/Colic compared to AP/Pain and AP/Occlusion. In these latter GCIs, contrast-enhanced examinations and higher $\mathrm{kV}(110 / 120 \mathrm{kV})$ were mostly used. These findings could be related to the fact that $\mathrm{CT}$ for AP/Colic is commonly performed in low dose thanks to the high natural contrast between kidney stones and tissues. The higher values observed for AP/Occlusion could be related to the high percentage of FBP reconstruction (50\%) when compared to other GCIs. However, the low number of examinations (18) in this subgroup limits the significance of this difference. A small but statistically significant difference in $\mathrm{CTDI}_{\mathrm{vol}}$, respectively DLP, was observed between Head/Hemo, respectively Head/Tumour, and other GCIs in head examinations. However the small gap in dose indices between head GCIs tends to indicate 
that similar protocols are used whatever the indication. The same interpretation stands for CAP examinations where differences between two GCIs are moderate and statistically nonsignificant. Indeed, for these body parts, more homogeneous practices were noticed in $\mathrm{kV}$ use (mostly 110/120 kV) and modulated mA (Table 3).

NDRLs implemented in many countries $[15,18,19,21]$ are only based on an anatomic region, for single CT acquisition. Few studies involved the influence of patient size [19] or clinical indication $[14,16,17,20,22]$. The impact of patient size on radiation dose was well established in the United States of America (USA) recent study on their NDRLs [19]. Nevertheless, patient grouping was done in terms of water equivalent diameter for body examination and lateral thickness for head examination. These settings are not easily accessible for local benchmarking neither for national assessment. Moreover, USA NDRLs were developed based on examination type not on clinical indication. NDRLs were proposed in few studies for few clinical indications and for standard sized patients [14, 16, 17, 20, 22]. These studies showed that patient radiation dose depends on the number of CT acquisitions within same examination and on image quality requirements for diagnosis. Recently, the European Commission launched a tender project on clinical DRLs (EUCLID - European Study on Clinical Diagnostic Reference Levels for X-ray Medical Imaging [26]). The project is led by the European Society of Radiology and started in August 2017. The aim of this project is to provide up-to-date clinical DRLs for the most important, from a radiological perspective, $\mathrm{x}$-ray imaging tasks in Europe. The $75^{\text {th }}$ percentile dose indices for normalweight patients in this study were compared with those of other clinical NDRLs (Table 4). Our results were generally lower than other reported data or within the lowest values. One reason might be the evolution of techniques and professional practices within the time gap between different surveys. Modern CT scanners with dose-reduction options (current 
modulation and IR) were generally used in this study. Another possible explanation is that, with a few exceptions, this study relied on voluntary medical physicists. It seems reasonable to consider that facilities where a medical physicist is involved tend to have lower dose protocols. Proposed French NDRLs based on CT examinations grouped by anatomical regions were also indicated in Table 4. All CTDI ${ }_{\mathrm{vol}}$ and DLP values of this study were similar or lower than the proposed French NDRLs except for traumatic brain injury and CAP infectious foci examinations where DLP value was a little higher.

Morphology and clinical based patient dose indicators are useful to benchmark CT imaging protocols and optimise patient radiation dose as a function of image quality need. Twentyfifth, $50^{\text {th }}$ and $75^{\text {th }}$ percentiles (Tables $2 \mathrm{a}$ and $2 \mathrm{~b}$ ) of total examination DLP per $n_{a}$ value are provided to encourage facilities to optimize dose as low as reasonably achievable by taking into consideration the image quality. Ideally, facilities should analyse and compare their median, size-grouped dose indices by clinical indication with the respective clinical and size based $25^{\text {th }}, 50^{\text {th }}$ and $75^{\text {th }}$ percentiles values. If size-grouped dose indices are not available, facilities should compare their overall median indices with the "all Genders", "all BMIs" and/or "all acquisitions" $25^{\text {th }}, 50^{\text {th }}$ and $75^{\text {th }}$ percentiles values. The implementation of this optimisation process is most effective if the facility has a Dose Archiving and Communication System (DACS) that automatically monitors patient radiation dose indices.

This study comes with some limitations. First, no strict image quality analysis was carried out but the patient radiation dose data corresponded to examinations performed in the institutions according to their normal practice of image quality selection for the given indication. We have to assume that the vast majority of the collected examinations met image quality standards because we assume the vast majority were interpreted. The second limitation concerns the 
representativeness of this survey. First, a little number of CT scanners contributes to some GCIs (Head/Tumour, Chest/Infectious, Chest/Metastases and CAP/Infectious) resulting from grouping examinations by patient size and number of acquisitions within examination.

Second, less than $20 \%$ of the facilities were private centres. However, numerous general public hospitals (i.e. non-academic) share their equipment with the private sector in so-called “economic interest groups" regional organization (GIE for groupements d'intérêt économique). Third, this study relied on voluntary medical physicists involved in university imaging departments in France, who represent a small proportion of facilities in France. Therefore, the results of this pilot study cannot be considered as representative of the whole French radiological practice.

In addition, the recent ICRP recommendations [13] could not been generalised in establishing dose distributions for all sub-groups by using the median of each CT scanner. Nonetheless, the two calculation methods were tested and gave acceptable differences. Moreover, we accepted that no correction was applied to the recorded $\mathrm{CTDI}_{\mathrm{vol}}$ values. We assumed that $\mathrm{CTDI}_{\mathrm{vol}}$ values were in agreement with the national quality criteria (within $\pm 20 \%$ deviation) checked annually and after tube replacement. Lastly, the manual process for patient size data and examination dose information in some departments not using a DACS, led to missing data in some cases. Moreover, the use of an Excel sheet to manually collect the data limited the number of examinations compared to an automatic collection with dedicated software, such as in the US DRLs study [19]. However, the verification of the clinical indication was more feasible with the Excel sheet.

\section{Conclusion}

This study was, to our knowledge, the first national survey based on clinical indication and patient size and evaluating CT dose. Results provide a tool for CT dose optimisation that is 
better adapted to clinical practices and to the variability of patients' morphology. In order to capture continual future trends in CT technologies and practices evolution, this study should be periodically updated. This study could serve as an important input for the national radiation protection authorities to future updates of French DRLs after taking into account the study limitations.

\section{Acknowledgements}

The authors are very grateful to the SFPM (Société Française de Physique Médicale) for its support, to the SFR (Société Française de Radiologie) and its associated organ sections for their contribution in this study: CIREOL (Société francophone d'imagerie tête et cou), SFNR (Société Française de Neuroradiologie), SIAD (Société d'Imagerie Abdominale et Digestive), SIFEM (Société d'Imagerie de la Femme), SFICV (Société Française d'Imagerie Cardiovasculaire), SIGU (Société d'Imagerie Génito-Urinaire), SIMS (Société d'Imagerie Musculo-Squelettique), SIT (Société d'Imagerie Thoracique). The authors also wish to express their deepest gratitude to the staff (medical physicists, radiologists, technologists and radiation protection experts and officers) of the 53 radiology centres for their participation in this study and their efforts in providing the best data quality.

\section{Funding}

This research did not receive any specific grant from funding agencies in the public, commercial, or not-for-profit sectors. 


\section{References}

[1] Pearce MS, Salotti JA, Little MP, McHugh K, Lee C, Kim KP, et al. Radiation exposure from CT scans in childhood and subsequent risk of leukaemia and brain tumours: a retrospective cohort study. Lancet 2012;380:499-505. https://doi.org/10.1016/S01406736(12)60815-0.

[2] Mathews JD, Forsythe AV, Brady Z, Butler MW, Goergen SK, Byrnes GB, et al. Cancer risk in 680000 people exposed to computed tomography scans in childhood or adolescence: data linkage study of 11 million Australians. Br Med J 2013;346:f2360. https://doi.org/10.1136/bmj.f2360.

[3] Huang WY, Muo CH, Lin CY, Jen YM, Yang MH, Lin JC, et al. Paediatric head CT scan and subsequent risk of malignancy and benign brain tumour: a nation-wide populationbased cohort study. Br J Cancer 2014;110: 2354-60. https://doi.org/10.1038/bjc.2014.103.

[4] Krille L, Dreger S, Schindel R, Albrecht T, Asmussen M, Barkhausen J, et al. Risk of Cancer Incidence before the Age of 15 Years after Exposure to Ionising Radiation from Computed Tomography: Results from a German Cohort Study. Radiat Environ Biophys 2015;54:1-12. https://doi.org/10.1007/s00411-014-0580-3.

[5] Boice Jr. JD. Radiation Epidemiology and Recent Paediatric Computed Tomography Studies. Annals of the ICRP 2015;44: 236-48. https://doi.org/10.1177/0146645315575877.

[6] Journy N, Roué T, Cardis E, Ducou Le Pointe H, Brisse H, Chateil JF, et al. Childhood CT Scans and Cancer Risk: Impact of Predisposing Factors for Cancer on the Risk Estimates ». J Radiol Prot 2016;36: N1-7. https://doi.org/10.1088/0952-4746/36/1/N1.

[7] Meulepas JM, Ronckers CM, Merks J, Weijerman ME, Lubin JH, Hauptmann M. Confounding of the association between radiation exposure from CT scans and risk of leukemia and brain tumors by cancer susceptibility syndromes. J Radiol Prot 2016;36:953-74. https://doi.org/10.1088/0952-4746/36/4/953.

[8] International Commission on Radiological Protection. The 2007 recommendations of the International Commission on Radiological Protection. ICRP publication 103. Ann ICRP $2007 ; 37: 1-332$

[9] International Commission on Radiological Protection. Radiological protection and safety in medicine. ICRP publication 73. Ann ICRP 1996;26:1-47

[10] European Community. On health protection of individuals against the dangers of ionizing radiation in relation to medical exposure. Council directive 97/43 (Euratom). Official Journal of the European Communities 1997; 22-7 
[11] European Community. Laying down basic safety standards for protection against the dangers arising from exposure to ionising radiation. Council directive 2013/59 (Euratom). Official Journal of the European Union 2014;17.1.L13

[12] International Commission on Radiological Protection. Diagnostic reference levels in medical imaging: review and additional advice. Ann ICRP 2001;31:33-52

[13] International Commission on Radiological Protection. Diagnostic reference levels in medical imaging. ICRP Publication 135. Ann. ICRP 2017;46(1)

[14] Treier R, Aroua A, Verdun FR, Samara E, Stuessi A, Trueb PR. Patient doses in CT examinations in Switzerland: Implementation of national diagnostic reference levels. Radiat Prot Dosim 2010;42:244-54

[15] European Commission. Diagnostic Reference Levels in Thirty-six European Countries Part 2/2. Radiation Protection $n^{\circ} 180$. https://ec.europa.eu/energy/sites/ener/files/documents/RP180\%20part2.pdf; 2014 [accessed 19 March 2018].

[16] Public Health England. Doses from computed tomography (CT) examinations in the UK 2011 review. https://www.gov.uk/government/uploads/system/uploads/attachment_data/file/349188/PHE _CRCE_013.pdf; 2014 [accessed 19 March 2018].

[17] Sundhedsstyrelsen. Udviklingen i brug af røntgenundersøgelser i Danmark. https://www.sst.dk/da/straalebeskyttelse/roentgen/ /media/6BFF6AD1889B42AAAE71560 759BC2F3F.ashx; 2015 [accessed 19 March 2018].

[18] Roch P, Célier D, Dessaud C, Etard C. Using diagnostic reference levels to evaluate the improvement of patient dose optimisation and the influence of recent technologies in radiography and computed tomography. Eur J Radiol 2018;98:68-74. https://doi.org/10.1016/j.ejrad.2017.11.002.

[19] Kanal KM, Butler PF, Sengupta D, Bhargavan-Chatfield M, Coombs LP, Morin RL. U.S. Diagnostic Reference Levels and Achievable Doses for 10 Adult CT Examinations. Radiology 2017;284:120-33. https://doi.org/10.1148/radiol.2017161911.

[20] Health Canada. Canadian computed tomography survey - national diagnostic reference levels. http://www.healthycanadians.gc.ca/publications/security-securite/canadiancomputed-tomography-survey-2016-sondage-canadien-tomodensitometrie/alt/cct-surveysondage-ct-eng.pdf; 2016 [accessed 19 March 2018].

[21] Bundesamt für Strahlenschutz. Bekanntmachung der aktualisierten diagnostischen Referenzwerte für diagnostische und interventionelle Röntgenanwendungen. Vom 22 Juni 
2016. http://www.bfs.de/SharedDocs/Downloads/BfS/DE/fachinfo/ion/drw-roentgen [accessed 11 February 2019].

[22] Lajunen A. Indication-Based Diagnostic Reference Levels for Adult CT-Examinations in Finland. Radiat. Prot. Dosimetry 2015 Jul;165(1-4):95-97.

[23] International Electrotechnical Commission. Medical electrical equipment-part 2-44: particular requirements for the safety of X-ray equipment for computed tomography. Geneva, 2002.

[24] World Health Organisation. Physical status: the use and interpretation of anthropometry. Report of a WHO Expert Committee. WHO Technical Report Series 854. Geneva, 1995.

[25] Robinson MS, Bidmos MA (2009) The skull and humerus in the determination of sex: reliability of discriminant function equations. Forensic Sci Int 186:86.e1-86.e5. https://www.sciencedirect.com/science/article/pii/S0379073809000085. [accessed 12 February 2019].

[26] EUCLID - European Study on Clinical Diagnostic Reference Levels for X-ray Medical Imaging. Coordinated by the European Society of Radiology. http://www.eurosafeimaging.org/euclid [accessed 12 February 2019]. 


\section{Table legends:}

Table 1: List of 17 groups of clinical indications (GCIs). Number of centres, CT scanners and examinations $\left(\mathrm{n}_{\mathrm{e}}\right)$ included in the national survey. Description of clinical practices in terms of mean number of acquisitions per examination $\left(\left\langle\mathrm{n}_{\mathrm{a}}\right\rangle\right)$, percentage distribution of $\mathrm{n}_{\mathrm{a}}$ and contrast media use.

Table 2a: Number of CT examinations $\left(\mathrm{n}_{\mathrm{e}}\right)$ and total exam DLP range $\left(50^{\text {th }}\left(25^{\text {th }}-75^{\text {th }}\right.\right.$ percentile)) based on the number of acquisitions per examination $\left(\mathrm{n}_{\mathrm{a}}\right)$ and on patient's gender for 6 head and neck GCIs. Non-significant (--) sub-groups counting less than 20 examinations are not presented. Total exam DLP values are given for the 16-cm diameter phantom in head, ear and mandible/maxilla examinations and the 32-cm diameter in cervical spine examinations. p results of the Wilcoxon rank sum test between women and men values are presented $(\mathrm{p}<0.05$ are marked with $*)$.

Table 2b: Number of CT examinations $\left(n_{e}\right)$ and total exam DLP range $\left(50^{\text {th }}\left(25^{\text {th }}-75^{\text {th }}\right.\right.$ percentile)) based on the number of acquisitions per examination $\left(\mathrm{n}_{\mathrm{a}}\right)$ and on patient's BMI range for 11 trunk or full-body GCIs. Non-significant (--) sub-groups counting less than 20 examinations are not presented. Total exam DLP values are all given for the 32-cm diameter phantom, except for the Polytrauma GCI where the examination DLP value can be a sum of 16-cm and 32-cm DLP values for full-body examinations.

Table 3: Comparison of dosimetric indices and acquisition characteristics between different GCIs relative to the same body part. Only single-acquisition examinations are considered for all GCIs. Only normal BMI patients are considered for trunk and full-body examinations. DLP and $\mathrm{CTDI}_{\mathrm{vol}}$ values are given for the 16-cm diameter phantom in head, ear and mandible/maxilla examinations, 32-cm for other body parts. This analysis was not possible for Polytrauma GCI because of a non-significant number of single-acquisition examinations. 
* indicates a p-value $<0.05$ for every Wilcoxon rank sum tests calculated with other GCIs of the same body part.

Table 4: Comparison of this study's results with published international DRLs. Rounded values of the $75^{\text {th }}$ percentile of CTDI ${ }_{\mathrm{vol}}(\mathrm{mGy})$ and DLP $(\mathrm{mGy} \cdot \mathrm{cm})$ for normal-weight patients (except for head \& neck examination) are considered for this study. CTDI ${ }_{\mathrm{vol}}$ and DLP values are given for the 16-cm diameter phantom in head, ear and mandible/maxilla examinations, 32-cm for other body parts.

\section{Figure captions:}

Figure 1: Distribution of collected examinations by scanner manufacturer, commissioning year and GCI. The size of the dots is proportional to the number of examinations. Scanner manufacturer is colour coded.

Figure 2: Distributions of multi-acquisitions examination DLP for 17 studied GCIs in 9 anatomical regions in all BMIs patients. The boxes represent the inter-quartile range (IQR, distance between the $1^{\text {st }}$ and $3^{\text {rd }}$ quartiles), the black lines in between mark the median value. The upper (lower) whiskers extend to the largest (smallest) value no further than $1.5 \times \mathrm{IQR}$ from the boxes. Data falling outside the whiskers range are plotted individually as outliers. Figure 3: Distribution of CTDI $\mathrm{vol}_{\mathrm{vol}}(\mathrm{a}, \mathrm{b})$ and DLP values $(\mathrm{c}, \mathrm{d})$ for single-acquisition examination in Head, Thorax, AP and CAP GCIs. Only normal BMI patients are considered for trunk examinations. $\mathrm{CTDI}_{\mathrm{vol}}$ and DLP values are given for the 16-cm diameter phantom in head examinations, 32-cm for other body parts. The boxes represent the inter-quartile range (IQR, distance between the $1^{\text {st }}$ and $3^{\text {rd }}$ quartiles), the black lines in between mark the median value. The upper (lower) whiskers extend to the largest (smallest) value no further than $1.5 \times$ IQR from the boxes. Data falling outside the whiskers range are plotted individually as outliers. 
4000 -

हิ $3500-$

¿

吕 $2500-$

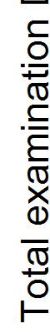
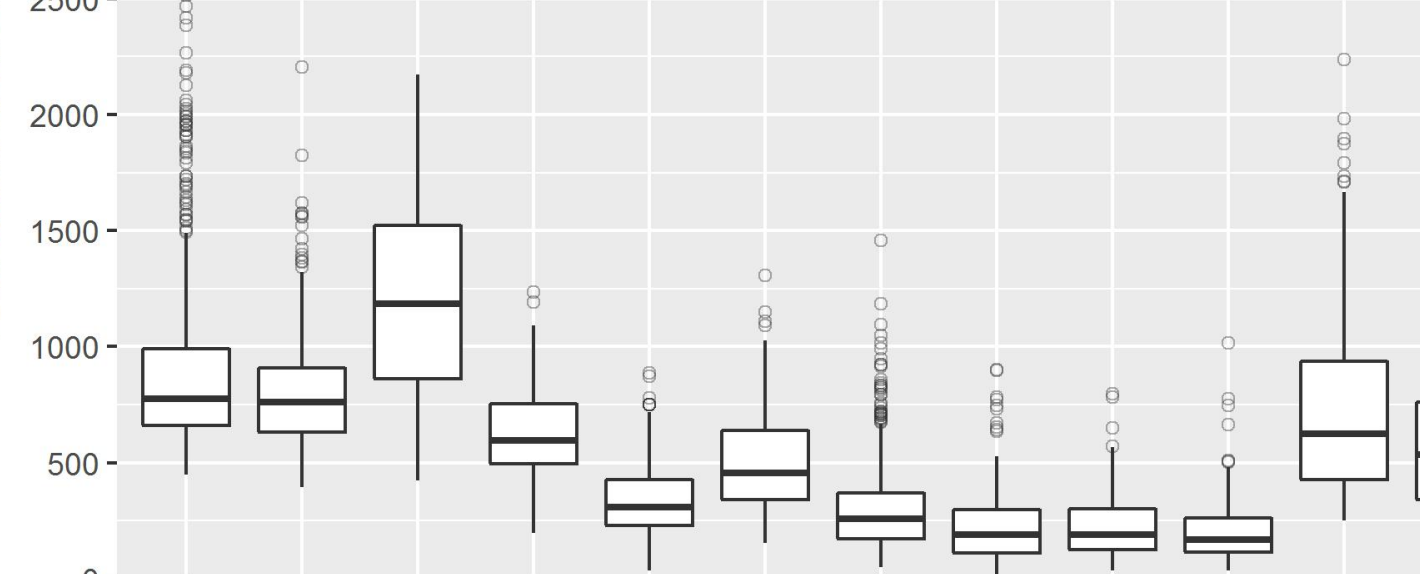


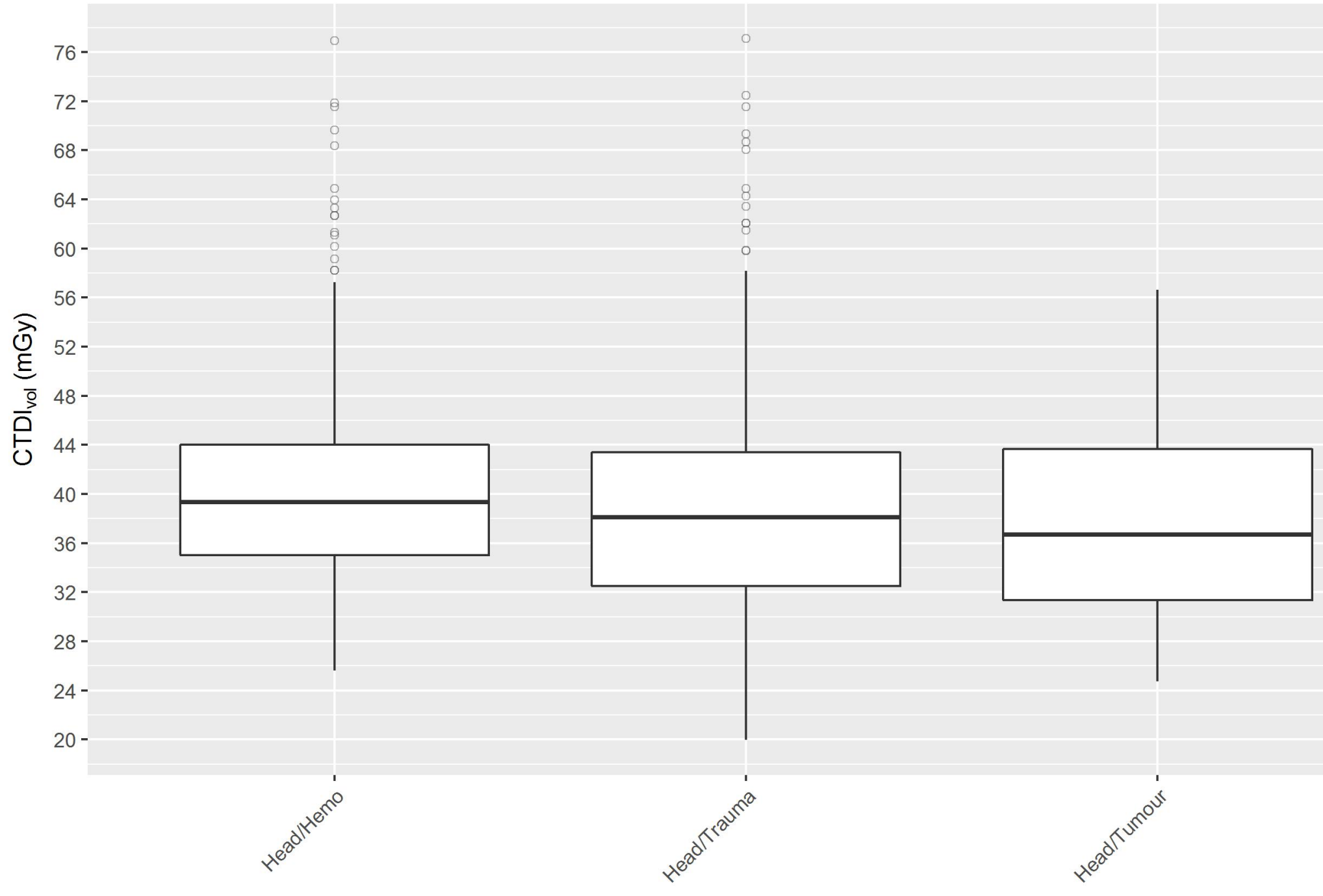




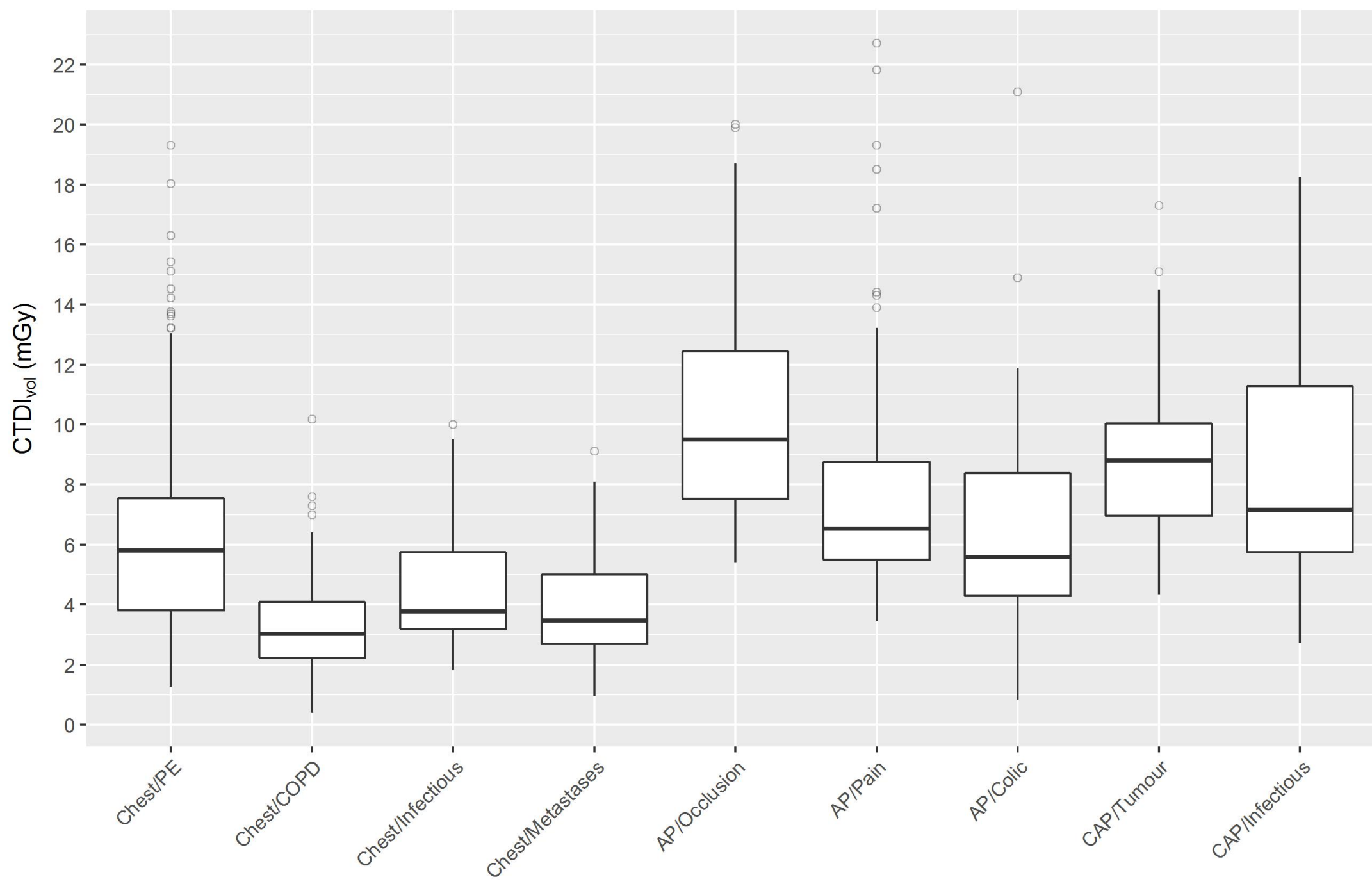




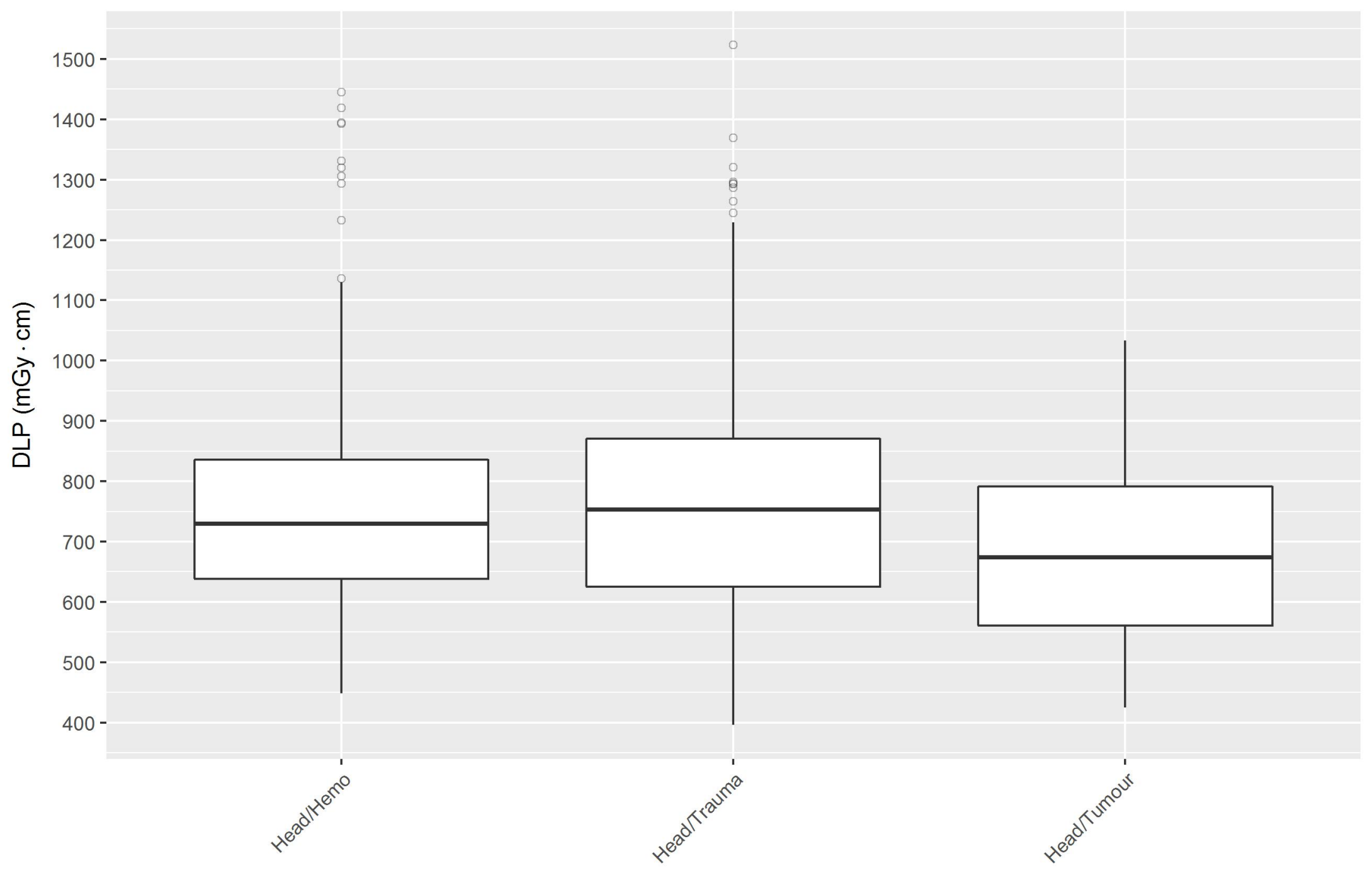




\section{Highlights}

- Patient morphology- and indication-based CT dose indices were assessed nationwide.

- Dose indices differed significantly between some indications in the same body region.

- For head \& neck examinations, dose indices were higher for male patients.

- For trunk examinations, dose indices increased consistently with patient BMI.

- This study provides a tool for CT dose optimisation adapted to clinical practices. 